\title{
L’agriculture biologique: controverses et enjeux globaux de développement en Afrique
}

\author{
Ludovic Temple ${ }^{1,3, *}$ et Hubert de Bon ${ }^{2,3}$ \\ ${ }^{1}$ CIRAD, UMR Innovation, 34398 Montpellier, France \\ 2 CIRAD, UPR HORTSYS, 34398 Montpellier, France \\ ${ }^{3}$ Univ Montpellier, Montpellier, France
}

\begin{abstract}
Résumé - L'agriculture biologique offre plusieurs options pour documenter les transitions technologiques vers de nouveaux modèles de production, même si elle présente des aspects controversés: faiblesse des rendements, accessibilité aux normes, valeurs des écobilans ou accroissement du travail. En mobilisant différentes situations en Afrique subsaharienne, ce numéro thématique des Cahiers Agricultures contribue à illustrer ces controverses. Les articles constitutifs montrent comment l'agriculture biologique définie par les normes des pays industriels ne peut rendre compte de la diversité des réalités agricoles africaines. Il s'ensuit l'émergence de nouvelles certifications et demandes des sociétés locales. Cette émergence reste contrainte par l'insuffisance des bases de connaissances comparatives des réalités productives entre l'agriculture biologique et conventionnelle. Des innovations méthodologiques pour réduire les asymétries de connaissances sur la comparaison des performances sont alors proposées. Les résultats interrogent la nécessité de nouveaux indicateurs intégrant les questions de sécurité nutritionnelle et sanitaire. Ils montrent que l'agriculture biologique peut aussi être un levier de l'accroissement des rendements quand la rente forestière a été consommée par l'agriculture d'exportation. Tout en éclairant les controverses, ce numéro thématique pose l'hypothèse, que sous certaines conditions, l'agriculture biologique est une opportunité de rupture de paradigme technologique qui répond aux enjeux de développement en Afrique. Il invite à ne pas confondre cette rupture avec les mécanismes de transition incrémentaux portés par l'agroécologie.
\end{abstract}

Mots clés : agriculture biologique / Afrique / innovation / méthode d'évaluation / développement

Abstract - Organic agriculture: Controversies and global development issues in Africa. Organic agriculture offers several options for documenting technological transitions towards new production models, although there are some controversies: low yields, access to standards, effective ecological balance, increased labour... By mobilizing different situations in sub-Saharan Africa, this thematic issue of Cahiers Agricultures provides information on these controversies. The presented papers illustrate how the organic agriculture defined by the standards of industrialized countries cannot reflect the diversity of African agricultural realities. As a result, the need for new certifications and applications for local companies is emerging. However, this emergence is constrained by the lack of comparative knowledge of productive realities between organic and conventional agriculture. Methodological innovations to reduce asymmetries in knowledge about performance comparison are then proposed. The results question the need for new indicators integrating nutrition and health security issues. They show that organic agriculture may also be a mean for increasing yields when the forest rent has been destroyed by export agriculture. While shedding light on the controversies, this thematic issue makes the hypothesis that, under certain conditions, organic agriculture is a breaking opportunity for a technological paradigm that meets the development issues in Africa. It invites us not to confuse this break with the mechanisms of agro-ecology-driven incremental transition.

Keywords: organic agriculture / Africa / innovation / evaluation / development

\footnotetext{
*Auteur de correspondance : ludovic.temple@cirad.fr
} 


\section{Introduction}

Le secteur agricole et alimentaire est particulièrement concerné par la convergence d'enjeux globaux : changement climatique, transition écologique, sécurité alimentaire et nutritionnelle (Faure et al., 2018), décarbonisation de l'industrie (Daviron, 2019). Ces enjeux renouvellent la demande sociétale sur l'urgence de changer le paradigme technologique dominant qui, depuis de nombreuses années, oriente les transformations de l'agriculture (Temple et al., 2018). Dans ce renouvellement, l'agriculture biologique (AB) propose plusieurs scénarios potentiellement complémentaires: une alternative pour la transition globale de l'agriculture mondiale (Muller et al., 2017; Wilbois et Schmidt, 2019), un levier d'activation des transitions écologiques de l'agriculture conventionnelle (AC) (Lamine, 2012), une niche d'opportunité pour des marchés de rente (Allaire, 2016). En partant du rôle de la recherche agronomique dans les processus d'innovation liés à l'AB en Afrique au sein de différents projets (Diversité des agricultures biologiques en Afrique subsaharienne et contribution à la sécurité alimentaire [ABASS], Projet Glofood, CIRAD-INRA ; Platform for Africa - European Partnership in Agricultural Research for Development [PAEPARD], Forum for Agricultural Research of Africa), cet article introduit un numéro thématique des Cahiers Agricultures qui présente 7 articles (Temple et De Bon, 2020). Les contributions de ce numéro approfondissent la connaissance de l'AB en Afrique sur trois plans : l'analyse de ses potentialités pour réaliser la sécurité alimentaire (Dury et al., 2017), la caractérisation des mécanismes de gouvernance institutionnelle qui structurent son développement, et enfin l'illustration de situations techniques qui expérimentent les conditions de durabilité d'une production biologique. D'un point de vue méthodologique, nous recourons à une analyse systémique qui conduit à alimenter des controverses en intégrant les résultats de différentes études de cas. Pour cela, dans une première section, nous explorons les connaissances bibliographiques qui critiquent la capacité de l'AB à structurer un changement de paradigme technologique qui réponde aux enjeux de développement. Dans une deuxième section, nous mettons en exergue les contributions des articles de ce numéro au renouvellement des connaissances et des méthodes sur les trajectoires technologiques de l'AB africaine. La conclusion discute de la façon dont ces renouvellements font progresser les futurs possibles de l'AB en Afrique.

\section{Controverses sur la capacité de l'agriculture biologique à répondre aux enjeux d'une sécurité alimentaire durable}

Les travaux qui contestent la capacité de l'AB à changer de paradigme technologique pour l'agriculture sont principalement regroupés dans les approches en termes de "land sparing 》 (Rundlöf et Smith, 2006; Smith, 2013 ; Kirchmann et al., 2016) et dans les scénarios prospectifs sur les demandes anticipées de biomasse agricole. Ces demandes sont liées à l'accroissement quantitatif des demandes alimentaires (transitions démographiques, transitions alimentaires dans les pays émergents ou moins avancés) ou à des demandes industrielles de la bio-économie ou de la production agricole pour l'énergie (Pahun et al., 2018). En relation avec cette demande quantitative, la globalisation du mode de production en $\mathrm{AB}$, en raison de ses faibles rendements (Van der Vossen, 2005; Reganold et Wachter, 2016; Lesur-Dumoulin et al., 2017; Seufert et al., 2019), pourrait conduire à étendre les superficies cultivées. Il en résulterait une destruction potentielle des réserves de biosphère ou, par la délocalisation des zones de production, des conséquences négatives sur le changement climatique (Searchinger et al., 2019). D'un point de vue écologique, il serait donc préférable de poursuivre un objectif d'accroissement de la productivité dont l'indicateur est le rendement agronomique (Bakehe, 2018), plutôt que des modes de production qui exigent un accroissement des superficies cultivées.

D'autres controverses sur les opportunités réelles que propose l'AB pour le développement sont analysées dans la littérature. Ainsi, par exemple, les normes d'AB sont pour l'instant principalement établies dans et par les pays industriels en fonction d'objectifs principaux d'amélioration de la qualité sanitaire des produits et de leur impact au regard de l'usage des intrants phytopharmaceutiques (Schwindenhammer, 2017). Ces normes restreignent l'usage de ces produits, ou inversement, dans certains cas, conduisent à l'usage de certains d'entre eux (Carimentrand, 2008). Ces normes participeraient également à orienter les firmes chimiques pour écologiser la fabrication d'intrants, c'est-à-dire vers la mise au point de pesticides biodégradables par une chimie doublement verte (Nieddu et Vivien, 2015; Bateman et al., 2018). Elles ne lient pas la conception des itinéraires techniques d'AB à un changement structurel des modes de production et de consommation. Elles tendent à piloter les innovations techniques sur des frontières technologiques pour optimiser une agriculture restant fortement tributaire de sa dépendance à des intrants issus des sources d'énergies fossiles et du secteur industriel. Les conséquences sur des enjeux environnementaux comme le changement climatique s'en retrouvent fortement discutées. Deux questions sont alors posées dans le contexte de l'agriculture africaine. Ces normes sont-elles adaptées à la réalité d'une agriculture qui est restée faiblement dépendante du secteur industriel? Comment diversifier leurs conditions de régulation pour faire reconnaître dans les modélisations prospectives (Dorin et Joly, 2019), la diversité des modes de production d'AB africaines qui structurent les transitions sociotechniques (Bui et al., 2016)?

Les externalités sociales et environnementales en termes d'écobilans de l'AB sont aussi débattues. En ce qui concerne les externalités sociales, il est commun de penser que l'AB, en produisant plus cher des produits de qualité, réserverait leur accès à des circuits commerciaux (rayons spécifiques, magasins spécialisés) dédiés aux catégories sociales à haut revenu. En réalité, cette critique est nuancée par des travaux sur les profils de consommateurs dans les pays industriels, qui montrent que les régimes alimentaires des consommateurs de produits biologiques étant plus frugaux, notamment en produits animaux, ils seraient accessibles à différents types de consommateurs malgré le différentiel de prix plus élevé observé produit par produit (Kesse-Guyot et al., 2018).

En ce qui concerne les écobilans, la reconversion biologique des approvisionnements de l'industrie agroalimentaire et de la grande distribution tend à industrialiser la production biologique alimentaire ou celle de matières 
premières biologiques (soja biologique pour l'élevage de porcs biologiques par exemple). Compte tenu de l'intensité en travail plus forte de l'AB, et du coût comparativement plus élevé du travail dans les pays de l'Organisation de coopération et de développement économiques (OCDE) que dans le reste du monde, l'approvisionnement en produits biologiques des acteurs industriels tend à se délocaliser dans des contextes socio-institutionnels où les règles $\mathrm{du}$ travail sont moins contraignantes. Cette délocalisation conforte, dans ces pays, un modèle économique de production industrielle qui pilote l'innovation technique par une demande de changements incrémentaux (choix de nouvelles molécules de protection de culture, fertilisants biologiques améliorés...). Cette délocalisation territoriale des approvisionnements accroît les coûts énergétiques et leur impact en termes de bilan carbone par rapport à des approvisionnements de proximité en produits qualifiés de «moins biologiques", car non certifiés AB. Des travaux montrent également que les ventes de produits biologiques en grandes surfaces classiques sont moins végétales et plus transformées qu'en magasins bio spécialisés, donc moins durables (Desquilbet et al., 2018).

Pour les pays africains, ces différentes controverses questionnent le scénario de faire de l'AB une opportunité de développement structurel en fonction de deux objectifs différents (Ministère de l'Économie et de la Planification du Territoire, République du Cameroun, 2018). Un premier objectif est de répondre à la demande d'approvisionnement industriel en produits biologiques des pays de l'OCDE. Cela pourrait permettre aux pays en développement de diversifier leurs exportations sur des produits à haute valeur ajoutée et une meilleure inclusion dans l'économie mondiale. La faiblesse des normes de biosécurité ou de leurs conditions d'application, le caractère incertain de la réglementation du travail et ses bas coûts actuels, les incertitudes juridiques sur la propriété des terres communautaires sont ainsi favorables à une délocalisation en Afrique de l'approvisionnement en produits biologiques des pays industriels. Cela interroge sur la nature des entreprises qui bénéficieront de ces délocalisations dans la captation de la valeur ainsi créée. Un deuxième objectif est ciblé sur les capacités de l'AB à approvisionner les marchés alimentaires locaux en produits de bonne qualité nutritionnelle et sanitaire. Cet objectif sous-entend le maintien d'un régime alimentaire frugal, sans suivre les scénarios mécaniques de transitions alimentaires globales que portent les pays industriels et les prospectives statistiques.

Dans un contexte intertropical où l'agriculture africaine reste majoritairement sur un mode de production familial et où les modèles de production de la révolution verte sont peu adoptés (Sourisseau et al., 2018), ces deux objectifs documentent des trajectoires technologiques d'AB différentes qui interagissent. La première est de spécifier une cohérence d'alternatives techniques au sein d'une trajectoire «d'intensification écologique » ou d'agroécologie générique (Seufert et al., 2019). L'AB est davantage considérée comme une alternative complémentaire d'une trajectoire d'intensification en intrants phytopharmaceutiques (Lotter, 2015). Elle permet alors d'en réduire l'intensité d'utilisation, de renouveler leur nature (bio-intrants) et affecte potentiellement peu les rendements comme on l'observe sur le coton au Paraguay (Silvie et al., 2010) ou dans certains vignobles en Europe (Muneret et al., 2018). Dans la deuxième option, l'AB revendique un affranchissement de la dépendance politique, économique et technologique de l'agriculture au secteur industriel. Elle porte une rupture de trajectoire technologique par la remise en cause du modèle productiviste agricole porté par les pays industriels. Elle conduit, sur le plan économique, à rechercher d'autres sources d'efficacité que les économies d'échelle ou les rendements, comme principalement les économies de gamme dans les systèmes de culture associées, les filières, les systèmes alimentaires (Temple et al., 2015). Les proximités existantes (organisationnelles, spatiales) entre consommateurs et producteurs deviennent alors des opportunités structurantes de ces nouvelles sources d'efficacité, au regard des besoins de sécurité alimentaire, nutritionnelle et sanitaire des populations locales (De Bon et al., 2018).

\section{Une contribution au renouvellement des connaissances et des méthodes documentant les futures trajectoires technologiques de l'agriculture biologique}

Ce numéro thématique des Cahiers Agricultures présente les résultats de projets de recherche dans différentes situations géographiques africaines (Cameroun, Côte d'Ivoire, Kenya, Mozambique, Ouganda).

Un premier axe de résultats analyse les connaissances sur les potentialités agronomiques de l'AB dans les situations africaines. En effet, d'une manière générale, les travaux scientifiques sur les rendements en $\mathrm{AB}$ sont rares en Afrique (Hauser et Lindtner, 2017; Adebiyi et al., 2020). Les données sont obtenues le plus souvent à partir de projets de développement, avec des bases très hétérogènes. L'article d'Andriamampianina et al. (2018) expérimente une méthode d'élicitation de dires d'experts pour produire de nouvelles informations à partir des bases de connaissances existantes. Il montre que les différences de rendement entre l'AC et l'AB ne sont pas homogènes suivant les filières et les localisations. Pour les fruits et légumes par exemple, l'article de De Bon et al. (2018) montre que les différentiels défavorables à l'AB sont beaucoup plus faibles que pour les productions céréalières et peuvent même s'inverser dans certains cas. De même, dans les zones où l'AC a détruit les bases naturelles de la fertilité au sens large, l'AB devient un moyen d'accroissement des rendements. Sur le plan méthodologique, ce constat pose la question de la comparaison des rendements par culture fondée sur des indicateurs d'homogénéité d'espèces, de variétés et de qualité, par rapport à des rendements au sein de cultures associées impliquant des espèces différentes avec des produits de qualité nutritionnelle variable.

Un deuxième axe de résultats met en exergue, dans l'article de Bendjebbar (2018), le rôle central des institutions dans la transformation des paradigmes socio-politiques pour reconnaître l'AB comme le levier d'une modernisation technologique de l'agriculture qui réponde aux attentes des marchés intérieurs. Deux éléments structurent cette évolution: les conditions d'émergence d'une solidification des normes de certification et les demandes sociétales des consommateurs du sud. Au Kenya, l'article de Tankam (2017) montre ainsi comment cette demande de produits, non certifiés du point de vue des normes internationales, se traduit de plus en plus fréquemment sur les marchés domestiques par des formes de 
certification «participatives », comme les Systèmes participatifs de garantie soutenus par l'Organisation des Nations unies pour l'alimentation et l'agriculture (FAO) ou par des systèmes de certification interne qui consolident l'émergence de normes nationales. Cette émergence présente des alternatives pour autonomiser le développement d'une $\mathrm{AB}$ destinée à l'approvisionnement des marchés locaux. Ainsi, au Cameroun, l'article de Bayiha et al. (2019) permet de bien préciser un ensemble de situations de production, de transformation et d'utilisation des productions agricoles et alimentaires (tubercules, plantains, produits alimentaires fermentés ou séchés), n'utilisant pas ou peu d'engrais chimiques ni de pesticides de synthèse, mais des intrants naturels. Ces situations peuvent structurer différents modèles de développement de l'AB en Afrique.

Ces travaux interpellent les normes actuelles de l'AB portées par les pays industriels, qui n'ont pas été élaborées pour accompagner le développement de l'AB à partir d'agricultures potentiellement biologiques. En effet, ces normes tendent à homogénéiser l'acceptation de l'AB au niveau mondial à partir de cahiers des charges techniques qui résultent de compromis sociétaux et entrepreneuriaux générés dans les pays où les marchés sont les plus grands. Elles apparaissent alors davantage comme un facteur de cantonnement de l'AB au sein de niches que comme un levier de déploiement et de reconnaissance de ce mode de production. Ainsi, les travaux présentés dans ce numéro thématique confirment que, en Afrique, l'AB repose sur des réalités agricoles qui existent depuis très longtemps, et dont les équilibres et les évolutions mobilisent des facteurs écologiques et sociaux localisés. Cette agriculture non (ou faiblement) certifiée renvoie à des situations que l'on retrouve dans des systèmes agroécologiques existants portés par l'agriculture familiale. Dans certains cas, comme le montre l'article de De Bon et al. (2019), la diversification vers d'autres cultures, comme l'anacardier sans intrant chimique en Côte d'Ivoire (Ruf et al., 2019), est un moyen de restaurer les rendements agricoles et la compétitivité des cultures d'exportation dans les zones cacaoyères d'agriculture familiale où la rente naturelle forestière a été épuisée.

Enfin, un dernier axe de résultats portant sur des expérimentations agronomiques concrètes explore différentes potentialités agroéconomiques d'itinéraires techniques recourant à des pratiques biologiques. Ainsi, en mobilisant un protocole expérimental rigoureux, Mukanga et al. (2019) démontrent qu'une modification de la technique de mise en place de l'arachide diminue significativement la teneur en aflatoxine et permet de respecter les normes sanitaires sans l'usage de pesticides. Cette contribution montre comment l'AB devient un lieu privilégié d'exploration et d'expérimentation coopérative entre la recherche et les agriculteurs.

\section{Conclusion}

La croissance rapide du marché mondial de produits biologiques (Willer et Lernoud, 2016) interpelle les institutions de recherche agronomique engagées dans l'accompagnement des transformations de l'agriculture dans les pays en développement. Cette croissance est nourrie par une demande de consommateurs de plus en plus sensibilisés à la qualité sanitaire (microbiologie et résidus de pesticides) des aliments
(Baudry et al., 2019) et plus particulièrement aux modes de production du point de vue des produits phyto-pharmaceutiques (Bellon et Penvern, 2014 ; Brureau et al., 2019). Cette croissance répond aussi à l'exigence d'accroître la résilience des systèmes alimentaires aux différentes instabilités climatiques et politiques (Dury et al., 2019). Elle est aussi liée à la demande citoyenne de prise en compte des impacts environnementaux et sociaux de l'agriculture, tant au Nord qu'au Sud (Branchet et al., 2018). La forme la plus visible de l'AB est encadrée par des cahiers des charges (standards) certifiés par tierce partie. En Afrique, cette $\mathrm{AB}$ certifiée concerne quelques produits échangés sur les marchés internationaux (cacao, café, coton, quinoa, riz, banane dessert, mangue, ananas), et parfois des produits destinés aux marchés nationaux (ignames, fruits et légumes) empruntant des circuits de distribution longs (François et al., 2005). Le renouvellement des objectifs des organismes internationaux pour la réduction des inégalités mondiales (environnementales, sociales) questionne en quoi l'AB pourrait être non plus une alternative parmi d'autres, mais un levier de transformation globale de l'agriculture. La capacité de l'AB à répondre aux enjeux de la sécurité alimentaire et de l'environnement spécifiques au continent africain est une condition à la réalisation de l'hypothèse précédente. Or cette capacité reste peu reconnue car peu connue (Kahane et al., 2013) au regard des controverses posées en introduction de cet article. Les spécificités (biophysiques, techniques, écologiques et sociales) africaines permettrontelles de nourrir une trajectoire technologique de l'agriculture mondiale en relation avec ce mode de production qui y reste dominant?

En ce qui concerne la controverse portant sur l'impact négatif d'une généralisation de l'AB sur la déforestation (land sparing), ce numéro spécial montre que les éléments méthodologiques qui fondent la comparaison d'efficacité entre l'AB et l'AC sont remis en cause. En effet, dans certaines situations, l'AB peut augmenter les rendements par rapport à l'AC. Cependant, les données mobilisables dans les pays africains sont insuffisantes pour des conclusions pertinentes au regard des potentialités d'accroissement des rendements actuels d'un certain nombre de productions sans forcément recourir au modèle de révolution verte. Par exemple, les nouvelles méthodes de multiplication de matériel végétal sur bananiers plantains permettent d'accroître significativement le rendement de cette culture vivrière (Kwa et Temple, 2019).

$\mathrm{Au}$-delà des rendements, un raisonnement en termes de productivité économique conduirait à analyser la réalité des coûts cachés qui accompagnent les stratégies de diffusion de l'AC dans les pays en développement. En l'occurrence, les travaux qui comparent les impacts de l'AB et de l'AC, notamment l'occupation des terres (déforestation), sont incomplets en termes d'impact. Sur le plan environnemental, ils ne tiennent pas compte des externalités sur la pollution des eaux, de l'air ou des effets de «débordement». Sur le plan social, ils n'analysent pas les conséquences de la concentration foncière, des exclusions sociales qui concentrent les populations dans les villes, accentuent les inégalités de patrimoine et globalisent des modes de vie urbains dont les impasses planétaires sont aujourd'hui connues. La validation croissante des causalités entre la consommation de l'AB et l'amélioration de la santé humaine justifie de prendre en compte la contribution de l'AB dans la diminution des coûts cachés que génère l'AC. Ainsi, les économies publiques (santé, environnement) que l'AB 
permet de générer sont à mieux révéler et à mieux prendre en considération.

Par ailleurs, ces scénarios reposent sur des hypothèses structurelles dans lesquelles les modèles alimentaires dominants de surconsommation de protéines animales, sucres et huiles, générateurs de maladies non transmissibles, se développent à l'échelle mondiale. De fait, le besoin d'accroissement de la biomasse agricole qui en découle ne peut qu'anticiper la performance d'une agriculture productiviste (Dorin et Joly, 2019). Or, dans les travaux rassemblés dans ce numéro, l'AB ne renvoie pas à la seule modification technique du mode de production, mais aussi à un changement complémentaire des modes de production, de distribution et de consommation des produits. Les situations identifiées en Afrique soulignent le maintien d'une agriculture vivrière insérée dans des circuits courts, structurée par l'autoproduction ou les réseaux de redistribution sociaux. Elles témoignent du fait que cette agriculture est à la fois inclusive et porteuse non pas d'archaïsmes, mais d'opportunités pour renouveler des formes d'articulation de la production et de la consommation, avec une agriculture moins intensive et plus multifonctionnelle.

Enfin, les travaux présentés dans ce numéro thématique montrent que les normes actuelles, dans leurs conditions de gouvernance et de mobilisation, sont pour l'agriculture africaine des contraintes à la reconnaissance d'une diversité des modes de production possible de l'AB. L'émergence de dispositifs de certification participatifs endogènes (Fouilleux et Loconto, 2017; Seufert et al., 2017; Lemeilleur et Allaire, 2018) et leur reconnaissance par les pays industriels sont des éléments clés d'une activation de l'AB comme un levier de changement de trajectoire technologique.

Les travaux rassemblés dans ce numéro soulignent l'insuffisance des bases de connaissances et d'informations sur les potentialités de l'AB africaine à répondre aux enjeux et urgences alimentaires existantes et futures. Ces travaux convergent aussi pour documenter la réalité de ces potentialités et de leurs conditions socio-politiques de réalisation. Ils confirment que la reconnaissance du mode de production en $\mathrm{AB}$ participe à un changement plus global de paradigme technologique agricole et alimentaire. Ils référencent des situations d'innovations concrètes qui montrent que les modes de production agricole et alimentaire et leurs connexions sont une variable centrale d'action pour rendre compatible des objectifs d'amélioration des conditions de vie humaine et des conditions d'existence à long terme de la vie biologique.

Remerciements. Nous remercions les évaluateurs ayant participé à l'amélioration de cet article.

\section{Références}

Adebiyi JA, Olabisi LS, Richardson R, Liverpool-Tasie LSO, Delate K. 2020. Drivers and constraints to the adoption of organic leafy vegetable production in Nigeria: a livelihood approach. Sustainability 12(1): 96 .

Allaire G. 2016. Que signifie le «développement» de l'AB? Innovations agronomiques 51: 1-17.
Andriamampianina L, Temple L, De Bon H, Malézieux E, Makowski D. 2018. Evaluation pluricritères de l'agriculture biologique en Afrique subsaharienne par élicitation probabiliste des connaissances d'experts. Cahiers Agricultures 27(4): 45002. DOI: 10.1051/ cagri/2018030.

Bakehe JP. 2018. Productivité agricole et déforestation dans le bassin du Congo. Economie rurale 366: 5-19.

Bateman ML, Day RK, Luke B, Edgington S, Kuhlmann U, Cock MJ. 2018. Assessment of potential biopesticide options for managing fall armyworm (Spodoptera frugiperda) in Africa. Journal of Applied Entomology 142(9): 805-819.

Baudry J, Pointereau P, Seconda L, Vidal R, Taupier-Letage B, Langevin B, et al. 2019. Improvement of diet sustainability with increased level of organic food in the diet: findings from the BioNutriNet cohort. The American Journal of Clinical Nutrition 109(4): 1173-1188.

Bayiha GP, Temple L, Mathe S, Nesme T. 2019. Typologie et perspective d'évolution de l'AB au Cameroun. Cahiers Agricultures 28: 3. DOI: $10.1051 /$ cagri/2019003.

Bellon S, Penvern S. 2014. Organic food and farming as a prototype for sustainable agricultures. In Bellon S, Penvern S, eds. Organic farming, prototype for sustainable agricultures. Dordrecht: Springer, pp. 1-19.

Bendjebbar P. 2018. La trajectoire d'institutionnalisation de l'agriculture biologique en Ouganda, success story de l'Afrique subsaharienne. Cahiers Agricultures 27(4): 45003. DOI: 10.1051/ cagri/2018029.

Branchet P, Cadot E, Fenet H, Sebag D, Ngatcha BN, BorrellEstupina V, et al. 2018. Polar pesticide contamination of an urban and peri-urban tropical watershed affected by agricultural activities (Yaoundé, Center Region, Cameroon). Environmental Science and Pollution Research 25(18): 17690-17715.

Brureau L, Emeville E, Helissey C, Thome JP, Multigner L, Blanchet P. 2019. Endocrine disrupting-chemicals and biochemical recurrence of prostate cancer after prostatectomy: A cohort study in Guadeloupe (French West Indies). International Journal of Cancer 146(3): 657-663. DOI: 10.1002/ijc.32287.

Bui S, Cardona A, Lamine C, Cerf M. 2016. Sustainability transitions: Insights on processes of niche-regime interaction and regime reconfiguration in agri-food systems. Journal of Rural Studies 48: 92-103.

Carimentrand A. 2008. Les enjeux de la certification biologique et équitable du quinoa (Chenopodium Quinoa Willd.) du consommateur au producteur. Thèse Économies et finances, Université de Versailles St Quentin en Yvelines. https://halshs.archives-ouvertes. fr/tel-01251410.

Daviron B. 2019. Biomasse : une histoire de richesse et de puissance. Versailles: Quae.

De Bon H, Temple L, Malézieux E, Bendjebbar P, Fouilleux E, Silvie P. 2018. L'agriculture biologique en Afrique: un levier d'innovations pour le développement agricole. Perspective 48. DOI: 10.19182/agritrop/00035.

De Bon H, Brun-Diallo L, Sène JM, Simon S, Sow MA. 2019. Rendements et pratiques des cultures maraîchères en agriculture biologique au Sénégal. Cahiers Agricultures 28: 2. DOI: 10.1051/ cagri/2019001.

Desquilbet M, Maigné E, Monier-Dilhan S. 2018. Organic food retailing and the conventionalisation debate. Ecological Economics 150: 194-203.

Dorin B, Joly PB. 2019. Modelling world agriculture as a learning machine? From mainstream models to Agribiom 1.0.Land Use Policy. DOI: 10.1016/j.landusepol.2018.09.028. 
Dury S, Vall E, Imbernon J. 2017. Production agricole et sécurité alimentaire en Afrique de 1'Ouest. Cahiers Agricultures 26(6): 61001. DOI: $10.1051 /$ cagri/2017047.

Dury S, Bendjebbar P, Hainzelin E, Giordano T, Bricas N, eds. 2019. Food systems at risk: new trends and challenges. Rome, Montpellier, Brussels: FAO, CIRAD and European Commission. DOI: 10.19182 /agritrop/00080.

Faure G, Chiffoleau Y, Goulet F, Temple L, Touzard JM. 2018. Renouveler les regards sur l'innovation dans les systèmes agricoles et alimentaires. In Faure G, Chiffoleau Y, Goulet F, Temple L, Touzard JM, eds. Innovation et développement dans les systèmes agricoles et alimentaires. Versailles: Quae, p. 5-16.

Fouilleux E, Loconto A. 2017. Voluntary standards, certification, and accreditation in the global organic agriculture field: A tripartite model of techno-politics. Agriculture and Human Values 34(1): $1-14$.

François M, Moreau R, Sylvander B. 2005. Agriculture biologique en Martinique. Expertise collégiale. Bondy: IRD Éditions, 304 p.

Hauser M, Lindtner M. 2017. Organic agriculture in post-war Uganda: Emergence of pioneer-led niches between 1986 and 1993. Renewable Agriculture and Food Systems 32(2):169-178.

Kahane R, Hodgkin T, Jaenicke H, Hoogendoorn C, Hermann M, Keatinge DJDH, et al. 2013. Agrobiodiversity for food security, health and income. Agronomy for Sustainable Development 33(4): 671-693.

Kesse-Guyot E, Baudry J, Allès B, Péneau S, Touvier M, Méjean C, et al. 2018. Déterminants et corrélats de la consommation d'aliments issus de l'agriculture biologique. Résultats du projet BioNutriNet. Cahiers de nutrition et de diététique 53(1): 43-52.

Kirchmann H, Kätterer T, Bergström L, Börjesson G, Bolinder MA. 2016. Flaws and criteria for design and evaluation of comparative organic and conventional cropping systems. Field Crops Research 186: 99-106.

Kwa M, Temple L. 2019. Le bananier plantain. Enjeux socioéconomiques et techniques, expériences en Afrique intertropicale. Versailles: Quæ, CTA, Presses agronomiques de Gembloux, $84 \mathrm{p}$.

Lamine C. 2012. Changer de système: une analyse des transitions vers l'agriculture biologique à l'échelle des systèmes agrialimentaires territoriaux. Terrains \& travaux 1: 139-156.

Lemeilleur S, Allaire G. 2018. Système participatif de garantie dans les labels du mouvement de l'agriculture biologique. Une réappropriation des communs intellectuels. Économie rurale 365: 7-27.

Lesur-Dumoulin C, Malézieux E, Ben-Ari T, Langlais C, Makowski D. 2017. Lower average yields but similar yield variability in organic versus conventional horticulture. A meta-analysis. Agronomy for Sustainable Development 37(5). DOI: 10.1007/ s13593-017-0455-5.

Lotter D. 2015. Facing food insecurity in Africa: Why, after 30 years of work in organic agriculture. I am promoting the use of synthetic fertilizers and herbicides in small-scale staple crop production. Agriculture and Human Values 32(1): 111-118.

Ministère de l'Économie et de la Planification du Territoire, République du Cameroun. 2018. Note d'analyse prospective: l'agriculture biologique, l'agriculture de demain? République du Cameroun, Yaoundé: Minepat, $18 \mathrm{p}$.

Muller A, Schader C, El-Hage Scialabba N, Brüggemann J, Isensee A, Erb KH, et al. 2017. Strategies for feeding the world more sustainably with organic agriculture. Nature Communications 8(1). DOI: $10.1038 / \mathrm{s} 41467-017-01410-w$.
Mukanga M, Matumba L, Makwenda B, Alfred S, Sakala W, Kanenga $\mathrm{K}$, et al. 2019. Participatory evaluation of groundnut planting methods for pre-harvest aflatoxin management in Eastern Province of Zambia. Cahiers Agricultures 28: 1. DOI: 10.1051/cagri/2019002.

Muneret L, Thiéry D, Joubard B, Rusch A. 2018. Deployment of organic farming at a landscape scale maintains low pest infestation and high crop productivity levels in vineyards. Journal of Applied Ecology 55(3): 1516-1525.

Nieddu M, Vivien FD. 2015. La chimie verte, une fausse rupture ? Les trajectoires de la transition écologique. In: Pahun J, Fouilleux, ed. Revue française de socio-économie 2: 139-153.

Pahun J, Fouilleux È, Daviron B. 2018. De quoi la bioéconomie estelle le nom ? Genèse d'un nouveau référentiel d'action publique. Natures Sciences Sociétés 26(1): 3-16. https://doi.org/10.1051/nss/ 2018020.

Reganold JP, Wachter JM. 2016. Organic agriculture in the twentyfirst century. Nature Plants 2(2): 15221.

Ruf F, Kone S, Bebo B. 2019. Le boom de l'anacarde en Côte d'Ivoire : transition écologique et sociale des systèmes à base de coton et de cacao. Cahiers Agricultures 28: 21. DOI: 10.1051/cagri/2019019.

Rundlöf M, Smith HG. 2006. The effect of organic farming on butterfly diversity depends on landscape context. Journal of Applied Ecology 43(6): 1121-1127.

Schwindenhammer S. 2017. Global organic agriculture policymaking through standards as an organizational field: When institutional dynamics meet entrepreneurs. Journal of European Public Policy 24(11): 1678-1697.

Searchinger TD, Wirsenius S, Beringer T, Dumas P. 2019. Publisher correction: Assessing the efficiency of changes in land use for mitigating climate change. Nature 565(7740): E9.

Seufert V, Ramankutty N, Mayerhofer T. 2017. What is this thing called organic? How organic farming is codified in regulations. Food Policy 68: 10-20.

Seufert V, Mehrabi Z, Gabriel D, Benton TG. 2019. Current and potential contributions of organic agriculture to diversification of the food production system. In: Agroecosystem diversity. Academic Press, pp. 435-452.

Silvie P, Martin J, Debru J, Vaissayre M. 2010. Le coton biologique au Paraguay. 2. Production et contraintes agronomiques. Biotechnologie, agronomie, société et environnement 14(2): 311-320.

Smith P. 2013. Delivering food security without increasing pressure on land. Global Food Security 2(1): 18-23.

Sourisseau JM, Bosc PM, Bonnal P, Bélières JF, Gasselin P, Valette E. 2018. In Bosc PM, Sourisseau JM, Bonnal V, Gasselin P, Valette E, Bélières JF, eds. Diversity of family farming around the World. Existence, transformations and possible futures of family farms. Springer, pp. 1-9.

Tankam C. 2017. L'organisation des marchés de producteurs de fruits et légumes biologiques à Nairobi, Kenya. Cahiers Agricultures 26 (3): 35006. DOI: 10.1051/cagri/2017020.

Temple L, De Bon H. 2020. Agriculture biologique en Afrique : diversité des trajectoires. Cahiers Agricultures Numéro thématique. https:// www.cahiersagricultures.fr/fr/component/toc/?task=topic\&id=795.

Temple L, Touzard JM, Boyer J, Requier-Desjardins D. 2015. Comparaison des trajectoires d'innovation pour la sécurisation alimentaire des pays du Sud. Biotechnologie, agronomie, société et environnement 19(1): 53-61. http://popups.ulg.ac.be/1780-4507/ index.php?id=11824.

Temple L, Chiffoleau Y, Touzard JM. 2018. Une histoire de l'innovation et de ses usages dans l'agriculture. In: Faure G, 
Chiffoleau Y, Goulet F, Temple L, Touzard JM, eds. Innovation et développement dans les systèmes agricoles et alimentaires. Versailles: Quae, pp. 19-37.

Van der Vossen HAM. 2005. A critical analysis of the agronomic and economic sustainability of organic coffee production. Experimental Agriculture 41(4): 449-473.
Wilbois KP, Schmidt J. 2019. Reframing the debate surrounding the yield gap between organic and conventional farming. Agronomy 9 (2): 82. https://doi.org/10.3390/agronomy9020082.

Willer H, Lernoud J. 2016. The world of organic agriculture. Statistics and emerging trends 2016. Research Institute of Organic Agriculture FiBL and IFOAM Organics International, $336 \mathrm{p}$.

Citation de l'article : Temple L, de Bon H. 2020. L'agriculture biologique : controverses et enjeux globaux de développement en Afrique. Cah. Agric. 29: 3. 